\title{
Physicochemical Parameters for Biological and Synthetic Surfactants Used as Detergents
}

Patrícia C. Bernardes, Emiliane A. Araújo, Marcia C. T. R. Vidigal, Nélio J. Andrade, Luis A. Minim and Valéria P. R. Minim

Department of Food Technology, Viçosa Federal University, Viçosa 36570-000, Minas Gerais, Brazil

Received: April 12, 2012 / Published: July 20, 2012.

\begin{abstract}
Biological and synthetic surfactants were compared in terms of their ability to reduce interfacial tension, change the thermodynamic characteristics of a pre-conditioned surface, and to modify the rheological properties of their respective formulations at two different temperatures. Both classes of surfactants were able to reduce the interfacial tension of their formulations to a similar level. However, the biosurfactants were more effective than the synthetics surfactants. Biosurfactants also altered the surface properties of stainless steel, rendering it hydrophilic. Microbial adhesion to stainless steel conditioned with biosurfactants was found to be thermodynamically unfavorable for all microbial strains tested. A linear relationship between shear stress and shear rate was obtained across a range of experimental conditions for all surfactant mixtures, indicating that all formulations behaved as Newtonian fluids.
\end{abstract}

Key words: Surfactants, biosurfactants, interfacial tension, microbial adhesion, stainless steel.

\section{Introduction}

The contamination of surfaces by spoilage and pathogenic microorganisms is a concern in the food industry. The development of biofilms in food processing environments results in product spoilage and possible risks to public health, in addition to create a number of serious problems for industrial fluid processing operations [1-3]. The presence of organisms such as Salmonella spp., Listeria monocytogenes and Escherichia coli in food processing environments can be a persistent source of contamination [4].

To reduce or eliminate microorganisms found on food contact surfaces, cleaning and disinfection procedures using physical and chemical methods have been extensively used over the years [5]. An interesting strategy is the pre-treatment of surfaces using surface-active compounds, also known as surfactants.

Surfactants can be classified into two main groups: synthetic surfactants and biosurfactants. Synthetic

Corresponding author: Nélio J. Andrade, D.S., researcher, research field: food hygiene. E-mail: nandrade@ufv.br. surfactants are produced by organic chemical reactions, whereas biosurfactants are produced by biological processes, being excreted extracellularly by microorganisms such as bacteria, fungi, and yeast [6]. Biosurfactants are produced using relatively cheap hydrophilic and hydrophobic substrates such as carbohydrates, vegetable oils, or even waste from the food industry [7]. When compared to synthetic surfactants, biosurfactants have several advantages, including high biodegradability, low toxicity, low irritancy, and compatibility with human skin $[8,9]$. Because of these superior properties, biosurfactants have many potential uses in the food, pharmaceutical, and cosmetic industries [10].

Some of the most extensively studied biosurfactants are the rhamnolipids, primarily produced by Pseudomonas aeruginosa [11]. The surfactant properties of a rhamnolipid mixture depend on its composition. This factor, in turn, varies according to the bacterial strain, culture conditions, and medium composition used for rhamnolipid production [12]. 
Nitschke et al. [13] investigated the effect of the biosurfactant surfactin on the adhesion of the food pathogens Listeria monocytogenes, Enterobacter sakazakii and Salmonella Enteritidis to stainless steel and polypropylene surfaces. The pre-conditioning of stainless steel surfaces with surfactin caused a reduction in the number of adhered cells of Enterobacter sakazakii and Listeria monocytogenes. The most significant result was obtained with L. monocytogenes, where the number of adhered cells was reduced by $10^{2}$ $\mathrm{CFU} / \mathrm{cm}^{2}$. On polypropylene surfaces, surfactin significantly decreased the adhesion of all strains.

In the literature, there are no references that describe the thermodynamic properties of surfaces that have been pre-conditioned with surfactants. This work aimed to compare biological and synthetic surfactants in terms of their ability to reduce interfacial tension, change the hydrophobicity of pre-conditioned surface and alter the rheological properties of their respective formulations at two different temperatures.

\section{Materials and Methods}

\subsection{Materials}

A rhamnolipid biosurfactant, consisting of a mixture of mono- and di-rhamnolipids from the glycolipid group produced by Pseudomonas aeruginosa was obtained from Jeneil Biosurfactant Co. (Saukville, Winconsin, USA) [14]. The molecular formulas for mono- and di-rhamnolipids are $\mathrm{C}_{26} \mathrm{H}_{48} \mathrm{O}_{9}$ and $\mathrm{C}_{32} \mathrm{H}_{58} \mathrm{O}_{13}$, respectively (Fig. 1). The critical micelle concentration (CMC) was found to be $0.035 \mathrm{~g} / \mathrm{L}$ by conductivity measurements performed at various dilutions [15].

The synthetic surfactant used was dodecylbenzene sodium sulfonate, and its $\mathrm{CMC}$ is $0.4 \mathrm{~g} / \mathrm{L}$.

\subsection{Bacterial Strains}

Staphylococcus aureus ATCC 6538, Escherichia coli ATCC 11229 Listeria innocua ATCC 33090, Pseudomonas aeruginosa ATCC 15442 and Bacillus cereus, isolated from pasteurized milk and identified as Ribo 1222173 S1 [16], were used throughout this work.
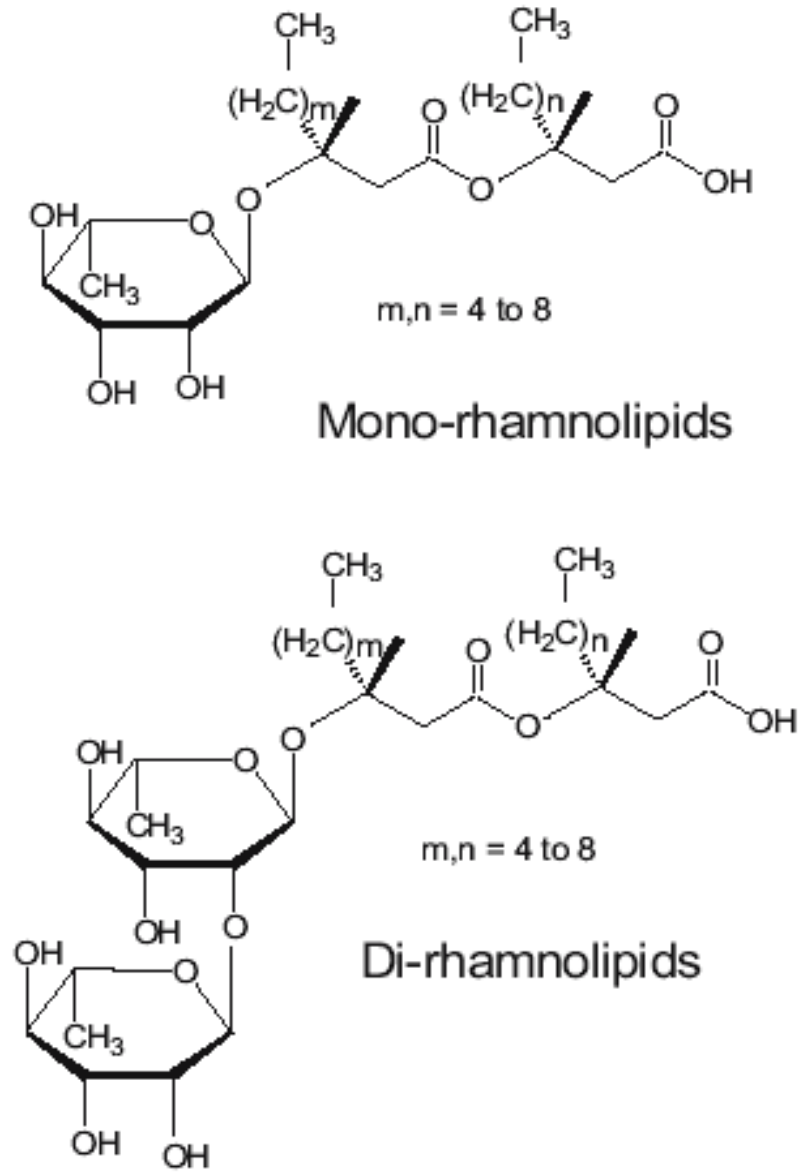

Fig. 1 Chemical structures of mono- and di-rhamnolipids.

\subsection{Detergent Formulations}

The detergent formulations used in the following experiments are shown in Table 1. The sodium carbonate concentration was selected to match the concentration typically used in the food industry for cleaning procedures. The concentrations chosen for other surfactants were above their respective CMCs to ensure the formation of micelles.

\subsection{Interfacial Tension Measurements of the Detergent Formulations}

The interfacial tension of each detergent formulation was measured by the pendant drop method using a goniometer (DSA 100, Kruss ${ }^{\circledR}$, Germany). The surface tension measurement was carried out at $25 \pm 1^{\circ} \mathrm{C}$. To calibrate the instrument, the surface tension of pure distilled Milli-Q water (Millipore, Illinois, USA) was measured before each set of experiments. 
Table 1 Different formulations for the treatment of stainless steel coupons.

\begin{tabular}{|c|c|}
\hline Formulations & Composition \\
\hline A & Sodium carbonate $(0.5 \%)$ \\
\hline B & $\begin{array}{l}\text { Sodium carbonate }(0.5 \%) \text { and dodecyl benzene } \\
\text { sodium sulfonate }(0.2 \%)\end{array}$ \\
\hline $\mathrm{C}$ & $\begin{array}{l}\text { Sodium carbonate }(0.5 \%) \text { and rhamnolipid } \\
(0.004 \%)\end{array}$ \\
\hline $\mathrm{D}$ & $\begin{array}{l}\text { Sodium carbonate }(0.5 \%) \text { and rhamnolipid } \\
(0.02 \%)\end{array}$ \\
\hline Control & Distilled water \\
\hline
\end{tabular}

2.5 Stainless Steel Conditioning with Detergent Formulations and Surface Hydrophobicity Measurements

Coupons $(10 \mathrm{~mm} \times 10 \mathrm{~mm} \times 0.05 \mathrm{~mm})$ of stainless steel \#4 were first cleaned by washing with a neutral liquid detergent and water, followed by rinsing with distilled water. Subsequently, they were put in a muffle furnace for $2 \mathrm{~h}$ at $200{ }^{\circ} \mathrm{C}$ to remove organic materials. After that, the coupons were immersed in detergent formulations (Table 1) for $24 \mathrm{~h}$ at $20-25{ }^{\circ} \mathrm{C}$. Control coupons without treatment were maintained in distilled water under the same conditions.

Surface hydrophobicity was determined based on contact angle measurements of the stainless steel with water, formamide and $\alpha$-bromonaphthalene using a goniometer. The total Gibbs free energy of interaction $\left(\Delta \mathrm{G}_{\mathrm{sws}}{ }^{\text {TOT }}\right)$ and the total free energy of adhesion between stainless steel and different bacteria $\left(\Delta \mathrm{G}_{\text {adhesion }}\right)$ were determined by appropriate formulas [17].

\subsection{Hydrophobicity of the Bacterial Strains}

One milliliter of each bacterial culture was stored at $-80{ }^{\circ} \mathrm{C}$ in a nutrient broth (Merck, São Paulo, Brazil) containing glycerol (80:20). A working culture of each strain, containing approximately $10^{7} \mathrm{CFU} / \mathrm{mL}$, was prepared by inoculating $10 \mathrm{~mL}$ of Brain Hearth Infusion (BHI, Merck, São Paulo, Brazil) with $100 \mu \mathrm{L}$ of frozen culture, followed by incubation at $32{ }^{\circ} \mathrm{C}$ for $24 \mathrm{~h}$. The culture was sub-cultured three times before use. The number of microorganisms in each suspension was obtained by total count using Plate Count Agar (PCA, Merck, São Paulo, Brazil) at $32{ }^{\circ} \mathrm{C}$ for $24 \mathrm{~h}$.
Contact angles for microorganism surfaces were determined on a layer of vegetative cells using the drop method [18]. First, the strains were activated twice in BHI broth (Merck, São Paulo, Brazil), creating suspensions with approximately $10^{7} \mathrm{CFU} / \mathrm{mL}$. The suspensions were centrifuged at $12,000 \mathrm{~g}$ for $10 \mathrm{~min}$ and washed three times in $0.1 \mathrm{M}$ phosphate buffered saline (PBS). The cell pellet was suspended in the same buffer, and the suspension was filtered using a cellulose acetate membrane $(0.45 \mu \mathrm{m}$ pore size, $27 \mathrm{~mm}$ in diameter, Millipore, Brazil) with negative pressure. During the filtration, $30 \mathrm{~mL}$ of pure water (Milli-Q) was added. The membranes were transferred to Petri plates containing $1 \%(\mathrm{v} / \mathrm{v})$ of agar and $10 \%(\mathrm{v} / \mathrm{v})$ of glycerol. The membranes were cut into three parts for contact angle measurements.

Each surface was classified as hydrophobic or hydrophilic according to the determination of the total free energy of interaction $\left(\Delta \mathrm{G}_{\text {sws }}{ }^{\mathrm{TOT}}\right)$. The total free energy of interaction among molecules of a surface (s) immersed in water $(\mathrm{w})$ was determined by the sum of the apolar and polar free energies of interaction, $\Delta \mathrm{G}_{\text {sws }}{ }^{\mathrm{LW}}$ and $\Delta \mathrm{G}_{\mathrm{sws}}{ }^{\mathrm{AB}}$, respectively:

$$
\begin{gathered}
\Delta G_{s w s}^{T O T}=\Delta G_{s w s}^{L W}+\Delta G_{s w s}^{A B} \\
\Delta G_{s w s}^{L W}=-2 \sqrt{\gamma_{s}^{L W}-\gamma_{w}^{L W}} \\
\Delta G_{s w s}^{A B}=-4\left(\sqrt{\gamma_{s}^{+} \gamma_{s}^{-}}+\sqrt{\gamma_{w}^{+} \gamma_{w}^{-}}-\sqrt{\gamma_{s}^{+} \gamma_{w}^{-}}-\sqrt{\gamma_{w}^{+} \gamma_{s}^{-}}\right)(3)
\end{gathered}
$$

Here, $\gamma^{\text {LW }}$ is the interfacial tension arising from Lifshitz-van der Waals forces; $\gamma^{+}$is the interfacial tension arising from the electron acceptor component of the acid-base interaction; $\gamma^{-}$is the interfacial tension of the electron donor component of the acid-base interaction; and $s$ and $w$ indicate surface and water, respectively [17].

When $\Delta \mathrm{G}_{\text {sws }}{ }^{\text {TOT }}>0$, the surface is considered hydrophilic; conversely, if $\Delta \mathrm{G}_{\mathrm{sws}}$ TOT $<0$, then the surface is considered hydrophobic.

Bacteria were used to determine the total Gibbs free energy of adhesion ( $\left.\Delta \mathrm{G}_{\text {adhesion }}\right)$ between conditioned stainless steel and each strain. 
2.7. Determination of the Total Gibbs Free Energy of Adhesion ( $\left.\Delta G_{\text {adhesion }}\right)$

From the values of the individual interfacial tension components, it is possible to determine the $\Delta \mathrm{G}_{\text {adhesion }}$ between two surfaces: microbial cells (b) and food processing surfaces (s):

$$
\begin{gathered}
\gamma_{b s}=\gamma_{b s}^{L W}+\gamma_{b s}^{A B} \\
\gamma_{b s}^{L W}=\gamma_{b}^{L W}+\gamma_{s}^{L W}-2 \sqrt{\gamma_{b}^{L W} \gamma_{s}^{L W}} \\
\gamma_{b s}^{A B}=2\left(\sqrt{\gamma_{b}^{+} \gamma_{b}^{-}}+\sqrt{\gamma_{s}^{+}} \gamma_{s}^{-}-\sqrt{\gamma_{b}^{+} \gamma_{s}^{-}}-\sqrt{\gamma_{b}^{-}} \gamma_{s}^{+}\right.
\end{gathered}
$$

Because the free energy is related to the interfacial tension, $\Delta \mathrm{G}_{\text {adhesion }}$ can be represented by the following:

$$
\begin{gathered}
\Delta G_{\text {adhesion }}=\Delta G_{b l s}^{L W}+\Delta G_{b l s}^{A B} \\
\Delta G_{b l s}^{L W}=\gamma_{b s}^{L W}-\gamma_{b l}^{L W}-\gamma_{s l}^{L W} \\
\Delta G_{b l s}^{A B}=\gamma_{b s}^{A B}-\gamma_{b l}^{A B}-\gamma_{s l}^{A B}
\end{gathered}
$$

Here, $\gamma_{\text {bs }}$ is the interfacial tension between the bacterial surfaces and the adhesion surface; $\gamma_{b l}$ is the interfacial tension between the bacterial surfaces and the liquid; and $\gamma_{\mathrm{sl}}$ is the interfacial tension between the adhesion surfaces and the liquid.

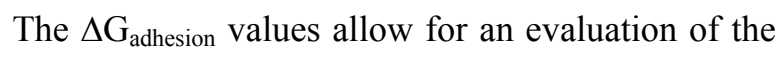
thermodynamics of the adhesion process: if $\Delta \mathrm{G}_{\text {adhesion }}<$ 0 , then the process is favorable; if $\Delta \mathrm{G}_{\text {adhesion }}>0$, then the process is unfavorable.

All measurements were performed in duplicate with three repetitions.

\subsection{Rheological Measurements of the Detergent Formulations}

Rheological measurements were performed using a Modular Advanced Rheometer System (HAAKE MARS, Thermo Electron Corp., Germany). The experiments were carried out at $25{ }^{\circ} \mathrm{C}$ and $70{ }^{\circ} \mathrm{C}$ for the different surfactant formulations, which correspond to manual cleaning temperatures and cleaning-in-place temperatures, respectively.

Steady rheological measurements were performed with double gap cylinder system (DG41) geometry, using a gap size set to $0.8 \mathrm{~mm}$. Flow curves were evaluated by measuring the shear rates from 0 to $1,200 \mathrm{~s}^{-1}$. N ew samples were used for measurements at each experimental temperature. Rheological parameter models were calculated from data using ThermoHaake RheoWin Pro 297 Software by the least squares method.

\section{Results and Discussion}

\subsection{Interfacial Tension of Detergent Formulations}

We observed that all surfactant formulations had interfacial tensions that were reduced relative to the control. However, formulations B, C and D produced greater reductions in interfacial tension than formulation A (Table 2). Although there is not a significant difference $(P>0.05)$ among the interfacial tensions of formulations B, C and D, biosurfactants are clearly advantageous as they are effective at low concentrations. It is important to emphasize that formulations $\mathrm{C}$ and $\mathrm{D}$ employed biosurfactant concentrations 50-fold and 10-fold lower, respectively, than the synthetic surfactant concentration in formulation B. Normally, sodium carbonate is used in the food industry to clean surfaces. In this work, we verified that the addition of a biosurfactant to a sodium carbonate formulation reduced its interfacial tension, thereby making the hygiene procedure easier and more efficient.

The results of this experiment were similar to the findings of Pornsunthorntawee et al. [6]. These researchers found that, in the case of the crude biosurfactant produced by Pseudomonas aeruginosa, the surface tension rapidly decreased as the concentration of biosurfactants increased. Furthermore,

Table 2 Interfacial tensions $(\mathrm{mN} / \mathrm{m})$ of different detergent formulations.

\begin{tabular}{ll}
\hline Formulations & Interfacial tension $(\mathrm{mN} / \mathrm{m})$ \\
\hline A & 61.07 \\
B & 28.22 \\
C & 28.44 \\
D & 28.09 \\
E & 72.10 \\
\hline
\end{tabular}


they found that a minimum surface tension of 29.0 $\mathrm{mN} / \mathrm{m}$ was obtained at an initial surfactant concentration greater than $0.02 \%$. In our experiment formulation $\mathrm{D}$ with a rhamnolipid concentration of $0.02 \%$ (Table 2) decreased the interfacial tension to $28.09 \mathrm{mN} / \mathrm{m}$ (46\%) when compared with formulation A.

\subsection{Hydrophobicity of Conditioned Stainless Steel Surfaces}

The conditioning of stainless steel changed its hydrophobicity. The coupons of stainless steel that were immersed in water (control formulation) were classified as hydrophobic because the $\Delta \mathrm{G}_{\mathrm{sws}}{ }^{\text {TOT }}$ value was negative, whereas coupons of stainless steel conditioned with the detergent formulations were considered hydrophilic $\left(\Delta \mathrm{G}_{\mathrm{sws}}{ }^{\text {TOT }}>0\right)$. In particular, treatment with a $0.004 \%$ formulation of rhamnolipid (Formulation $\mathrm{C}$ ) resulted in a more hydrophilic surface $\left(42.48 \mathrm{MJ} / \mathrm{m}^{2}\right)$ (Table 3).

According to thermodynamic principles, when $\Delta G_{s w s}^{T O T}$ is negative, there is a net cohesive attraction between the molecules of a surface when immersed in water. Thus, for a hydrophobic material, $\Delta G_{s w s}^{T O T}$ has a negative sign. In other words, molecules of a hydrophobic surface, when immersed in water, prefer to interact with each other rather than form an interface with water, whereas a positive value of $\Delta G_{s w s}^{T O T}$ shows that the molecules of a surface prefer to interact with adjacent water molecules rather than with themselves. Such a surface is hydrophilic [19].

The contact angle measurements are interpreted directly in terms of the surface energy of the adsorbed layer and provide access to equilibrium or steady-state adsorption because angle measurements do not perturb adsorption dynamics. The treatment of stainless steel with biosurfactants causes its surface properties to change [13]. The addition of a synthetic or biological surfactant alters the wettability of solids because of its adsorption at the solid/liquid interface.
Table 3 Values of the interfacial tension components $\left(\gamma^{L W}, \gamma^{+}\right.$and $\left.\gamma^{-}\right)$and the total Gibbs free energy of interaction $\left(\Delta \mathbf{G}_{\mathrm{sws}}{ }^{\text {TOT }}\right)$ for stainless steel surfaces conditioned with different detergent formulations.

\begin{tabular}{lllll}
\hline Formulations & $\gamma^{L W}$ & $\gamma^{+}$ & $\gamma^{-}$ & $\Delta \mathrm{G}_{\text {sws }}{ }^{\text {TOT }}\left(\mathrm{MJ} / \mathrm{m}^{2}\right)$ \\
\hline A & 28.53 & 1.39 & 51.25 & 31.75 \\
B & 27.16 & 4.93 & 37.20 & 11.30 \\
C & 28.73 & 1.15 & 60.68 & 42.48 \\
D & 28.36 & 3.01 & 59.80 & 34.60 \\
Control & 28.73 & 4.76 & 16.67 & -12.04 \\
\hline
\end{tabular}

Adsorption profiles suggest that the hydrocarbon chains of biosurfactants attach to hydrophobic surfaces and that their polar groups project toward aqueous solutions. This combination of events makes a surface more hydrophilic. The orientation of surfactant molecules at the solid/liquid interface determines the ability of the surfactant to improve the wetting ability of the liquid. A phenomenon involving a change in orientation of the adsorbed molecules could also be important in the kinetics of the wetting process [20].

\subsection{Total Gibbs Free Energy of Adhesion}

The adhesion process was thermodynamically favorable $\left(\Delta \mathrm{G}_{\text {adhesion }}<0\right)$ for all tested strains on stainless steel surfaces conditioned with formulation $\mathrm{B}$ or a control formulation (Table 4). In contrast, the adhesion process was unfavorable $\left(\Delta \mathrm{G}_{\text {adhesion }}>0\right)$ on stainless steel surfaces conditioned with formulations $\mathrm{C}$ or D. According to thermodynamic theory, adhesion is favorable only if the process results in a decrease in total free energy. Therefore, the rhamnolipid biosurfactant may potentially be incorporated into detergent formulations to reduce or avoid microbial adhesion and subsequent biofilm formation.

\subsection{Rheological Characteristics of the Detergent Formulations}

A linear relationship between shear stress and shear rate was obtained across a range of experimental conditions for all surfactant mixtures, indicating that all formulations behaved as Newtonian fluids (Figs. 2 and 3). Penott-Chang et al. [21] observed similar 
Table 4 Values of the total Gibbs free energy of adhesion $\left(\Delta \mathbf{G}_{\text {adhesion }}\right)$ between different bacteria and conditioned stainless steel surfaces.

\begin{tabular}{lrrrrl}
\hline & \multicolumn{5}{c}{ Total Gibbs free energy of adhesion $\left(\mathrm{MJ} / \mathrm{m}^{2}\right)$} \\
\cline { 2 - 6 } Bacterial strain & $\mathrm{A}$ & \multicolumn{1}{c}{$\mathrm{B}$} & \multicolumn{1}{c}{$\mathrm{C}$} & $\mathrm{D}$ & \multicolumn{1}{c}{ Control } \\
\hline S. aureus & 12.17 & -0.86 & 15.45 & 8.13 & -5.84 \\
E. coli & 9.46 & -1.37 & 11.82 & 5.08 & -3.50 \\
L. innocua & 4.20 & -6.05 & 6.51 & 0.15 & -8.40 \\
P. aeruginosa & 5.58 & -5.11 & 8.00 & 1.44 & -7.73 \\
B. cereus & 10.60 & -1.94 & 13.74 & 6.64 & -6.60 \\
\hline
\end{tabular}

Newtonian behavior while studying the rheology of aqueous solutions containing hydrophobically modified polyacrylamides and sodium dodecyl sulfate.

At a temperature of $25{ }^{\circ} \mathrm{C}$, surfactant formulations containing dodecyl benzene sodium sulfonate or diluted rhamnolipid $(0.004 \%)$ showed a reduction in viscosity compared to a solution containing only sodium carbonate. Table 5 shows the viscosity of all tested formulations at two temperatures $\left(25^{\circ} \mathrm{C}\right.$ and $70{ }^{\circ} \mathrm{C}$ ). The surfactant systems presented a low viscosity, i.e., comparable to the viscosity of water. According to Penott-Chang et al. [21], the presence of ionic groups in diluted solutions such as $\mathrm{B}$ and $\mathrm{C}$ promotes a reduction in viscosity. However, surfactant formulation $\mathrm{D}$ exhibited an increase in viscosity relative to the sodium carbonate control. The high concentration of rhamnolipid in this formulation $(0.02 \%)$ may have contributed to the formation of micelles, resulting in an increased viscosity for this solution.

As expected, an increase in temperature decreased the viscosity of all tested solutions. In an industrial context, a decreased viscosity can lead to more efficient cleaning procedures. More specifically, by decreasing the absolute viscosity $(\mu)$ at elevated temperatures, solvent flow across cleaning surfaces may be increased, thereby reducing pump power consumption and therefore energy costs.

\section{Conclusions}

Surfactant formulations containing low concentrations of rhamnolipids achieved reductions in interfacial tension comparable to a formulation containing a higher concentration of a synthetic surfactant. The pre-conditioning with biosurfactant converted microbial adhesion to stainless steel into a thermodynamically unfavorable process. Moreover, at

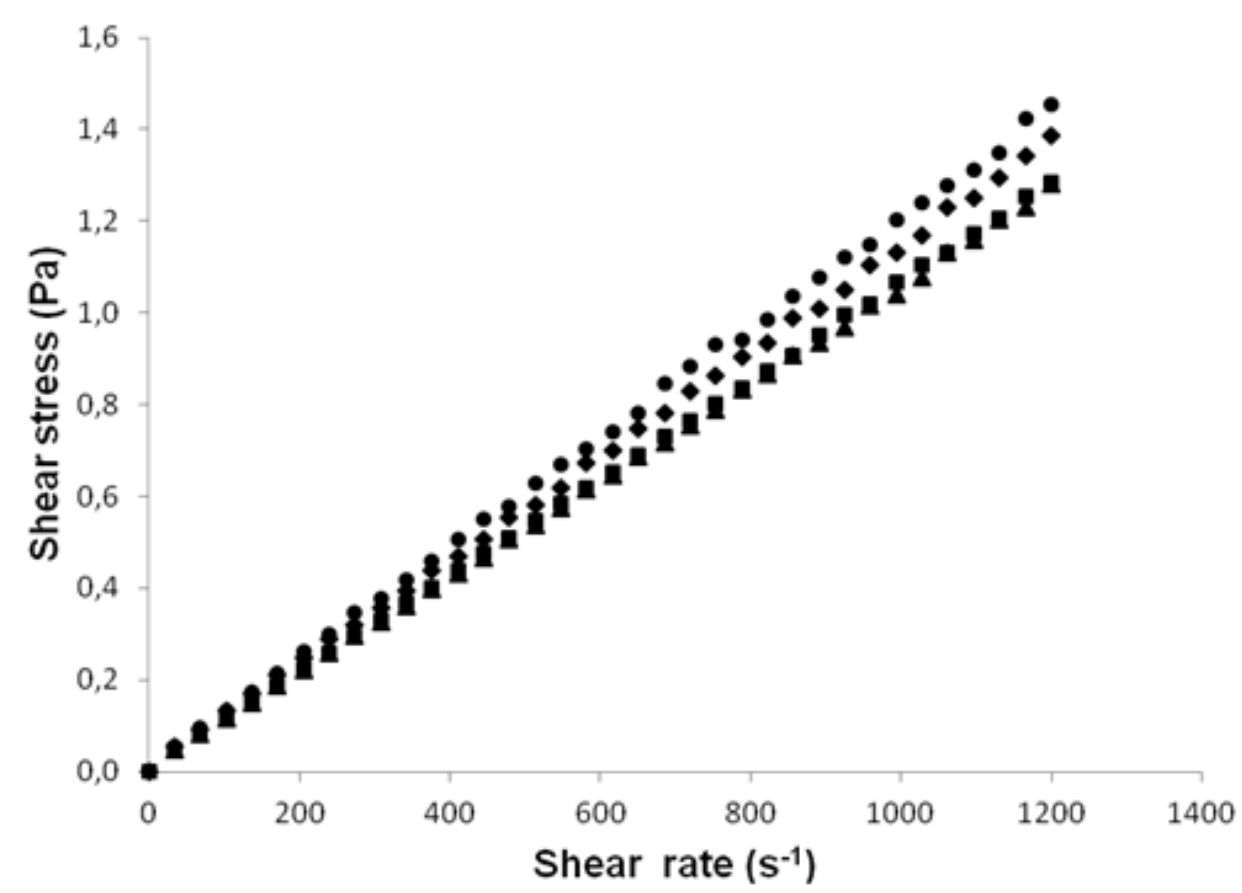

Fig. 2 Flow curves for different surfactant formulations at a temperature of $25{ }^{\circ} \mathrm{C}(\bullet$ Formulation A, $\square$ Formulation B, $\triangle$ Formulation C and $\bigcirc$ Formulation D). 


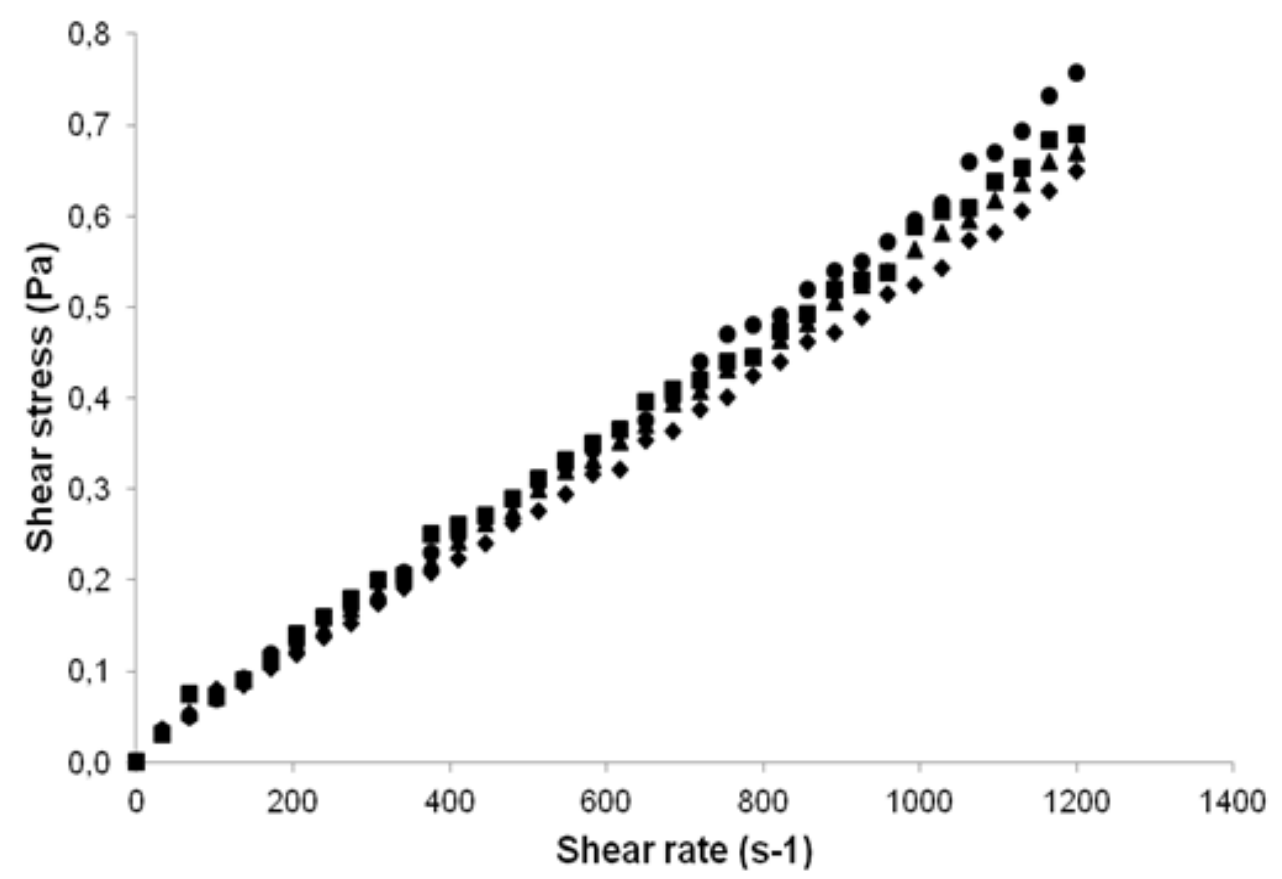

Fig. 3 Flow curves for different surfactant formulations at a temperature of $70{ }^{\circ} \mathrm{C}(\diamond$ Formulation $\mathrm{A}, \boldsymbol{\square}$ Formulation $\mathrm{B}, \boldsymbol{\Delta}$ Formulation C and $\bigcirc$ Formulation D).

Table 5 Rheological parameters of a Newtonian fluid model fitted to data from different surfactants at temperatures of 25 and $70{ }^{\circ} \mathrm{C}$.

\begin{tabular}{llll}
\hline Temperature $\left({ }^{\circ} \mathrm{C}\right)$ & Formulations & $\mu(\mathrm{MPa} \mathrm{s})$ & $\mathrm{R}^{2}$ \\
\hline \multirow{3}{*}{25} & $\mathrm{~A}$ & 1.146 & 0.999 \\
& $\mathrm{~B}$ & 1.068 & 0.999 \\
& $\mathrm{C}$ & 1.055 & 0.999 \\
& $\mathrm{D}$ & 1.210 & 0.999 \\
\hline \multirow{3}{*}{70} & $\mathrm{~A}$ & 0.536 & 0.999 \\
& $\mathrm{~B}$ & 0.577 & 0.958 \\
& $\mathrm{C}$ & 0.567 & 0.999 \\
& $\mathrm{D}$ & 0.614 & 0.973 \\
\hline
\end{tabular}

room temperature, a detergent formulation containing a low rhamnolipid concentration exhibited a reduced viscosity relative to a control. This formulation potentially offers a great advantage for cleaning procedures within the food industry. Therefore, a biosurfactant can be superior to a synthetic surfactant for preventing or delaying microbial colonization of food contact surfaces.

\section{Acknowledgments}

The authors thank the National Council for Scientific and Technological development $(\mathrm{CNPq} /$ Brazil) and the State of Minas Gerais
Foundation (FAPEMIG/ Brazil) for their financial support.

\section{References}

[1] G.T. Gunduz, G. Tuncel, Biofilm formation in an ice cream plant, A Van. Leeuw J. Microb. 89 (2006) 329.

[2] P.C. Calo-Mata, S. Arlindo, K. Boehme, T. Miguel, A. Pascoal, J.B. Velazquez, Current applications and future trends of lactic acid bacteria and their bacteriocins for the biopreservation of aquatic food products, Food Bio. Tech. 1 (2008) 43.

[3] S.P. Oliver, B.M. Jayarao, R.A. Almeida, Foodborne pathogens in milk and the dairy farm environment: Food safety and public health implications, Food Path Dis. 2 (2005) 115.

[4] A.C.S. Pires, N.F.F.Soares, L.H.M. Silva, N.J. Andrade, M.F.A.S. Silveira, A.F. Carvalho, Polydiacetylene as a biosensor fundamentals and applications in the food industry, Food Bio. Tech. 3 (2) (2008) 172.

[5] B. Joseph, S.K. Otta, I. Karunasagar, I. Karunasagar, Biofilm formation by Salmonella spp. on food contact surfaces and their sensitivity to sanitizers, International J. Food Microb. 64 (2001) 367.

[6] O. Pornsunthorntawee, P. Wongpanit, S. Chavadej, M. Abe, R. Rujiravanit, Structural and physicochemical characterization of crude biosurfactant produced by Pseudomonas aeruginosa SP4 isolated from petroleum-contaminated soil, Bioresource Technol. 99 (2008) 1589. 
[7] M. Nitschke, S.G.V.A.O. Costa, J. Contiero, Structure and applications of a rhamnolipid surfactant produced in soybean oil waste, Biotechnol. Progr. 21 (2005) 1593.

[8] I.M. Banat, R.S. Makkar, S.S. Cameotra, Potential commercial applications of microbial surfactants, Appl. Environ. Microb. 53 (2000) 495.

[9] S.S. Cameotra, R.S. Makkar, Recent applications of biosurfactantes as biological and immunological molecules, Curr. Opin. Microb. 7 (2004) 262.

[10] J.D. Desai, I.M. Banat, Microbial production of surfactants and their commercial potential, Microb. Mol. Biol. Rev. 6 (1997) 47.

[11] S. Lang, D. Wullbrandt, Rhamnose lipids—biosynthesis, microbial production and application potential, Appl. Microb. Biotech. 51 (1) (1999) 22.

[12] L. Guerra-Santos, O. Kapelli, A. Fiechter, Pseudomonas aeruginosa biosurfactant production in continuous culture with glucose as carbon source, Appl. Environ. Microb. 48 (1984) 302.

[13] M. Nitschke, L.V. Araújo, S.G.V.A.O. Costa, R.C. Pires, A.E. Zeraik, A.C.L.B. Fernandes, et al., Surfactin reduces the adhesion of food-borne pathogenic bacteria to solid surfaces, Lett. Appl. Microbiol. 49 (2009) 241.

[14] Jeneil Biosurfactant Co., LLC, Material Safety Data Sheet for JBR425 [Online], 2001, http://www.biosurfactant.com/downloads/jbr425msds.pdf.
[15] B. Dahr Azma, C.N. Mulligan, Extraction of copper from mining residues by rhamnolipids, Waste Manage 8 (3) (2004) 166.

[16] V.C. Salustiano, N.J. Andrade, N.F.F. Soares, J.C. Lima, P.C. Bernardes, L.M.P. Luiz, et al., Contamination of milk with Bacillus cereus by post-pasteurization surface exposure as evaluated by automated ribotyping, Food Control 20 (2009) 439.

[17] C.J. van Oss, Interfacial Forces in Aqueous Media, Marcel Dekker, Inc, New York, 1994.

[18] H.J. Busscher, A.H. Weerkamp, H.C. van Der Mei, A.W. van Pelt, H.P. de Jong, J. Arends, Measurement of the surface free energy of bacterial cell surface and its relevance for adhesion, Appl. Environ. Microbiol. 48 (5) (1984) 980.

[19] C.J. van Oss, R.F. Giese, The hydrophilicity and hydrophobicity of clay minerals, Clay Miner 43 (1995) 474.

[20] S.W. Musselman, S. Chander, Wetting and adsorption of acetylenic diol based nonionic surfactants on heterogeneous surfaces, Colloids Surfaces A 206 (2002) 497.

[21] E.K. Penott-Chang, L. Gouveia, I.J. Fernández, A.J. Muller, A. Díaz-Barros, A.E. Sáez, Rheology of aqueous solutions of hydrophobically modified polyacrylamides and surfactants, Colloids Surfaces A 295 (2007) 99. 